\title{
Transformationen der Antike in der zeitgenössischen Kunst
}

\author{
Christoph ZuschlaG
}

Das Gesamtverzeichnis 2007/08 des Verlages C. H. Beck ziert auf dem Umschlag die Arbeit Senza Titolo von Jannis Kounellis aus dem Jahr 1978 (Abb. 1). Es stellt sich die Frage, warum sich der Beck-Verlag mit seinen Bereichen Literatur, Sachbuch, Wissenschaft ausgerechnet mit diesem Werk präsentiert. Der Grund liegt für mich auf der Hand: Das Werk von Jannis Kounellis ist gleichsam doppelt codiert, es repräsentiert die Moderne und die Antike zugleich. Aber welche Antike eigentlich? Im Folgenden werde ich vier Werke unterschiedlicher Gattungen diskutieren, die zwischen 1975/76 und 2007 entstanden sind und auf ganz unterschiedliche Weise Gipsabgüsse antiker Skulpturen, also mechanisch hergestellte Reproduktionen, einbeziehen. Das Augenmerk liegt dabei auf folgenden Fragen: Auf welche Weise wird die Antike rezipiert, das heißt, wie wird der Gipsabguss formal verwendet und welche Rolle spielt er im neuen Werkkontext? Welche Vorstellungen von Antike manifestieren sich im Werk, welche Konstruktionen, Entwürfe und Bilder der Antike werden geschaffen? Welche neuen Sichtweisen auf die antiken Originale werden durch die zeitgenössischen Transformationen generiert und schließlich, welche Rolle spielen dabei medien- und gattungsspezifische Aspekte?

\section{Das verlorene Arkadien: Jannis Kounellis}

Eine intensive Antikenrezeption zeichnet das Werk einer Reihe italienischer Künstler aus, die der sogenannten »Arte povera« (wörtlich: arme oder ärmliche Kunst) zugerechnet werden. Der Begriff wurde 1967 von dem Kunstkritiker und

1 Vgl. zu den spezifischen Eigenschaften, dem künstlerischen Gebrauch und den Bedeutungen von Gips Uppenkamp (2002), zur Geschichte des Gipsabgusses Ladendorf (1958), 69-74, Berchtold (1987) und Cain (1995), zur Antikenrezeption in der bildenden Kunst im 20. Jahrhundert, speziell seit den 1970er Jahren, Unterdörfer (1998) und Krüger (2004). Eine grundlegende Neubewertung (nicht nur) des (Gips-)Abdrucks nimmt Didi-Huberman (1999) vor, der den Abguss, die Abformung, den Abdruck (empreinte) als Prozess, als Paradigma, als »ein konstitutives Modell für den allgemeinen Begriff des Bildes (36) ansieht. - Für konstruktive Anregungen in der Diskussion im Anschluss an den Vortrag danke ich Tatjana Bartsch, Horst Bredekamp, Johannes Myssok, Maximilian Schich, Martin Schieder und Peter Seiler, für wertvolle Hinweise und Ergänzungen zum Manuskript Marcus Becker. 


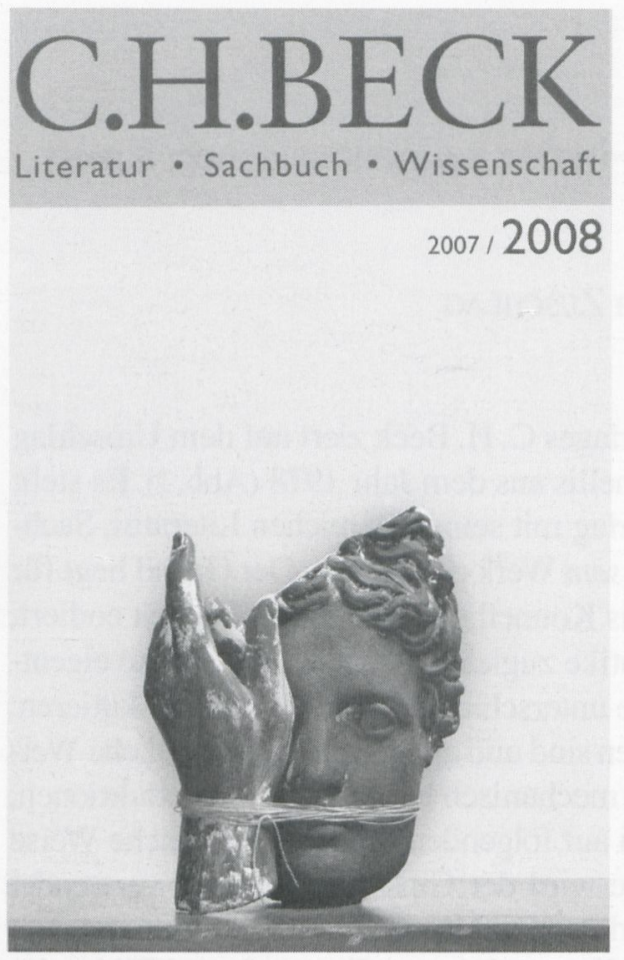

Abb. 1 Umschlag des C. H. Beck-Gesamtverzeichnisses 2007/08 mit Abbildung der Arbeit Senza Titolo, 1978, von Jannis Kounellis

Kurator Germano Celant geprägt, in Anlehnung an das »arme Theater« von Jerzy Grotowski. Einige Künstler der "Arte povera«, wie Giulio Paolini, Claudio Parmiggiani und eben Jannis Kounellis, verwendeten seit Mitte der 1960er Jahre zum Teil bis in die 1990er Jahre in ihren Montagen, Assemblagen, Objekten und Installationen Gipsabgüsse antiker Skulpturen.

So Kounellis, der 1936 in Piräus geboren wurde, in der eingangs erwähnten Arbeit Senza Titolo (Abb. 2). Das Objekt besteht aus zwei mit Kordel zusammengebundenen Fragmenten von Gipsabgüssen, nämlich einem schwarz gefassten Kopf und einer violett gefassten linken Hand. Es handelt sich um Gipsabgüsse römischer Marmorkopien griechischer Bronzeoriginale aus dem 4. Jahrhundert v. Chr.; die Hand stammt vom Hermes Farnese, der Kopf vom Apoll vom Belvedere, einer der berühmtesten Antiken überhaupt. Die Bildfindung von Kounellis erinnert, wie in der Forschung bereits erkannt wurde, an das Gemälde Le chant d'amour von Giorgio di Chirico aus dem Jahr 1914, in welchem der Kopf des Apoll vom Belvedere mit einem roten Gummihandschuh, einer Kugel und einer Lokomotive kombiniert ist. ${ }^{2}$ Hierzu schreibt Michaela Unterdörfer: »Der rätselhaften Verbindung des Götterkopfes mit dem banalen Gummihandschuh im Gemälde De Chiricos setzt Kounellis das klassische Maß des Menschen entgegen, das in dem Motiv von Kopf und Hand verdichtet ist. $\aleph^{3}$ Der in die Hand gestützte Kopf ist der klassische Melancholie-Gestus. ${ }^{4}$ Kounellis kombiniert versatzstückartig Fragmente von Gipsabgüssen zweier klassischer Bildwerke, bemalt den Gips grob und flüchtig, so dass das Weiß an verschiedenen Stellen durchscheint und

2 Giorgio de Chirico, Le chant d'amour, 1914, Öl auf Leinwand, 73 x 59,1 cm, New York, The Museum of Modern Art.

3 Unterdörfer (1998), 110.

4 Vgl. Melancholie. Genie und Wahnsinn in der Kunst (2005). 
Abb. 2 Jannis Kounellis, Senza Titolo, 1978, Gips, Deckfarben, Kordel, Eisensockel, 152 × 40 x $40 \mathrm{~cm}$, Hamburg, Hamburger Kunsthalle, Leihgabe aus Privatbesitz

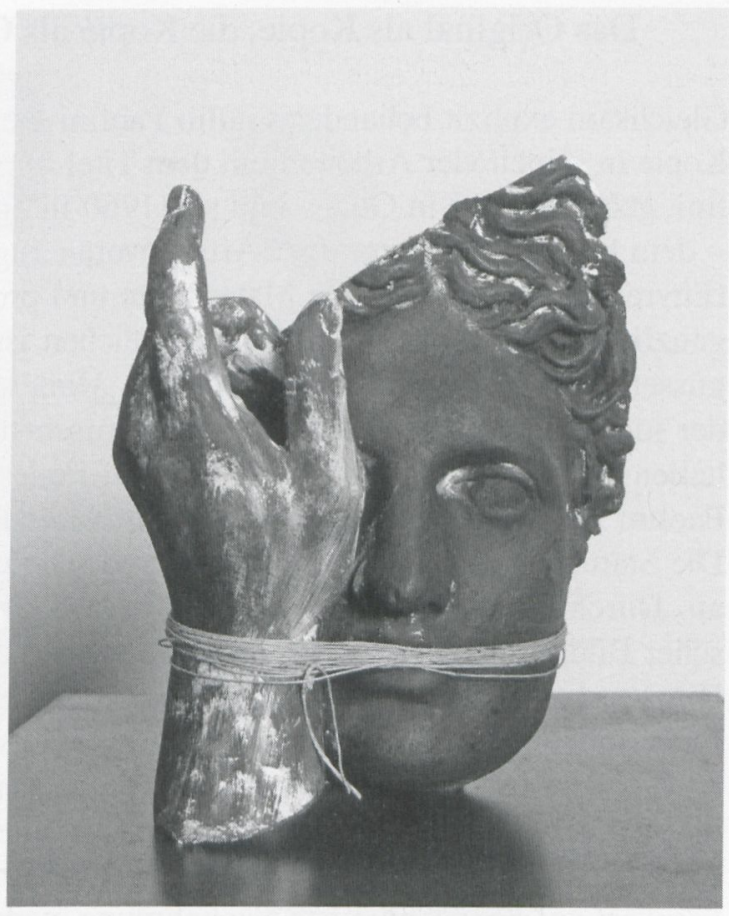

umwickelt die beiden Teile mehrfach mit einer dünnen Kordel. Das Ganze wirkt absichtsvoll roh, zugleich jedoch hochgradig ästhetisiert. Stephen Bann interpretiert die Verstümmelung und Fragmentisierung des Antikenabgusses bei Kounellis psychoanalytisch. Demnach enthalte Senza Titolo

[...] the imaginative challenge of what is neither intact nor perfect, and remains all the more powerful for not being manifested in its entirety. The eyes of Apollo are unseeing, and the finger of Hermes points nowhere. But the idea of an original plenitude of significance inherent in classical art, so integral a feature of Western visual culture ever since Winckelmann, seems not to have been entirely abandoned. ${ }^{5}$

Das Werk lässt sich somit als melancholisches Erinnerungsbild einer untergegangenen klassischen Kultur interpretieren, einer Kultur, in welcher nach Kounellis' Überzeugung die Moderne wurzelt. Der Künstler versteht sich dezidiert als europäischer, in der Antike verwurzelter Künstler. Seit über vierzig Jahren lebt er in Rom, umgeben von den Zeugnissen der Antike, auf die er sich in seinen Werken immer wieder bezieht. Cornelia Stabenow zufolge nimmt die Antike bei Kounellis »die Rolle eines verlorenen Arkadien ein. $\aleph^{6}$ 


\section{Das Original als Kopie, die Kopie als Original: Giulio Paolini}

Gleichsam explizit behandelt Giulio Paolini die Problematik von Original und Kopie in seinen vier Arbeiten mit dem Titel Mimesi von 1975/76 (Abb. 3). ${ }^{7}$ Paolini, geboren 1940 in Genua und seit 1960 in Turin tätig, wird - wie Kounellis - dem breiten Spektrum der »Arte povera « zugerechnet, obwohl er in seinem Euvre auf ärmliche, krude Materialien und provozierende Gesellschaftskritik gänzlich verzichtet. Paolinis Markenzeichen ist die Verwendung von Gipsabgüssen antiker Skulpturen. So auch in Mimesi. Zwei identische Gipsabgüsse der sogenannten Venus Medici, einer römischen Marmorkopie eines verlorenen hellenistischen Bronzeoriginals, sind auf Podesten einander gegenübergestellt. Paolini hat die Gipskopien formal nicht verändert und keine Farbe verwendet. Die Statuen stehen diagonal versetzt und sehen sich über die Schultern hinweg an. Durch dieses Auf-sich-selbst-bezogen-Sein des Werkes wird ein hermetischer Bildraum geschaffen, aus dem der Betrachter ausgeschlossen bleibt. Das Prinzip der Verdoppelung verweist auf die Reproduzierbarkeit künstlerischer Form, auf das Verschwinden des Originalitätsanspruchs in der Postmoderne und auf die fundamentale Problematik des Verhältnisses von Urbild und Abbild. In Mimesi demonstriert Paolini, dass alles Original und alles Kopie ist, diese Kategorien mithin ihre Gültigkeit verloren haben. Der Titel wiederum verweist auf das Prinzip der Nachahmung, wobei hier nicht die Natur, sondern die Kunst nachgeahmt wird!

Paolinis zentrales Thema, das Sehen, wird in Mimesi gleichsam explizit, indem das Sehen gezeigt wird. »Der Blick«, formuliert der Künstler im Begleittext zu Mimesi, »richtet sich auf sich selbst mit der Frage nach seiner eigenen Präsenz. $\ll^{8}$ Der Betrachter wird angehalten, seine visuellen Erfahrungen zu reflektieren. Paolini greift in seinen Werken mit Vorliebe auf klassisches Bildgut zurück, das er als Material betrachtet: »Das ,Material' wird als gültiger Formenvorrat verstanden, dessen sich der Künstler ebenso gut bedienen kann, wie wenn er sich die Natur zum Vorbild wählt. ${ }^{9}$ Doch liefert die Antike Paolini nicht nur ein schier unendliches Formenreservoir, aus dem er schöpfen kann, sondern vor allem auch die Möglichkeit der künstlerischen Selbstreflexion: »Antikenrezeption erscheint bei Paolini also als Teil einer europäischen Spielart von Konzeptkunst, die tautologische Konstruktionen ersinnt, in denen die Kunst über sich selbst meditiert. $\ll^{10}$

7 Vgl. hierzu Friedel (1984) und (1988), 95f., Unterdörfer (1998), 48f., Bätzner (2000), 92-96, sowie Krüger (2004), 39-63.

8 Giulio Paolini, zitiert nach Friedel (1984), 72.

9 Friedel (1984), 73.

10 Krüger (2004), 60. 


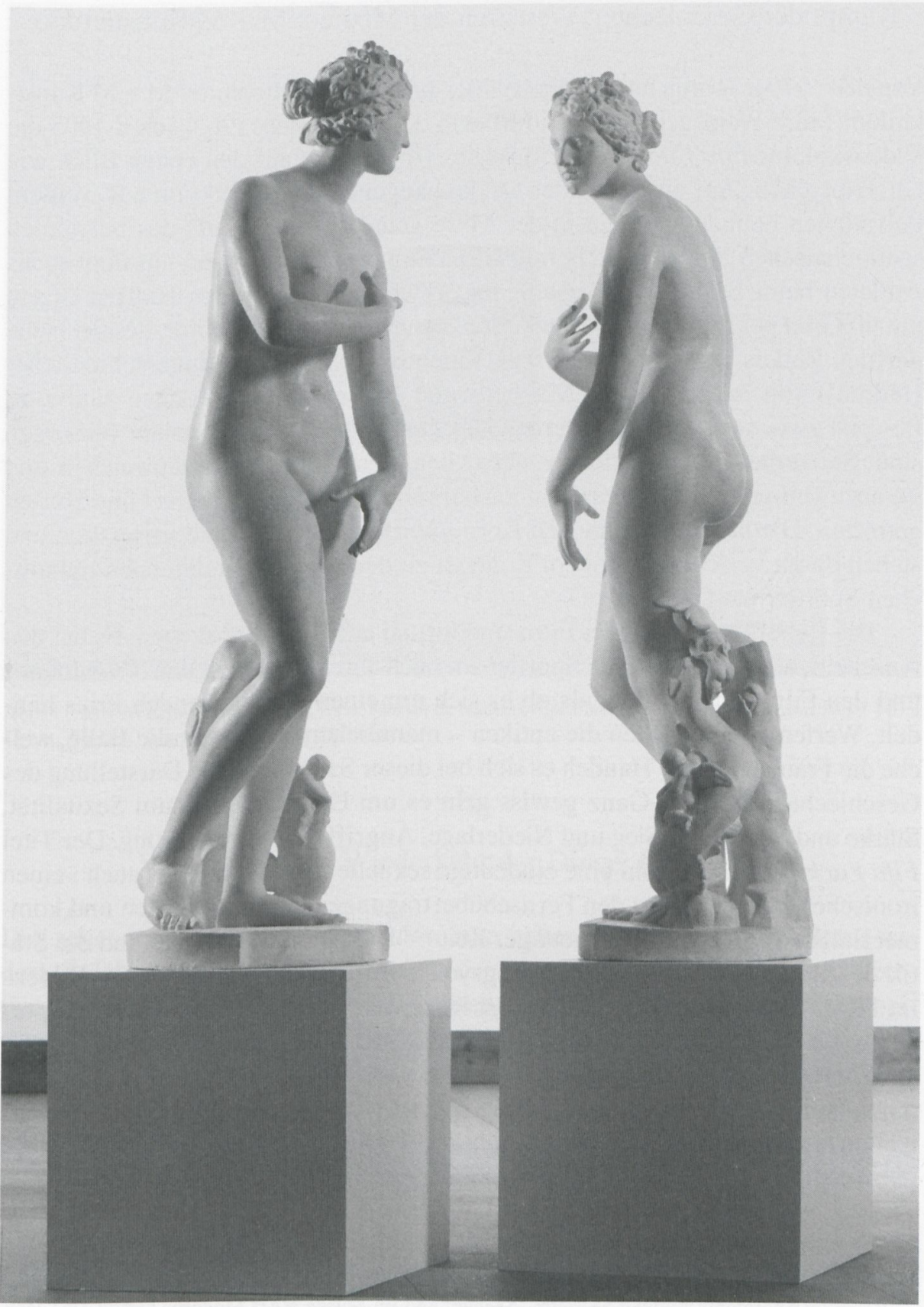

Abb. 3 Giulio Paolini, Mimesi, 1975/76, zwei Gipsabgüsse nach der Venus Medici, einschließlich Sockel jeweils 218 x 155 x $80 \mathrm{~cm}$, Berlin, Sammlung Paul Maenz 


\section{Kampf der Geschlechter, Wettstreit der Medien: M + M Künstlerduo}

Von den 1970er Jahren nun zu den 1990er Jahren. Das Münchner M + M Künstlerduo (Marc Weis, geb. 1965, und Martin de Mattia, geb. 1963) schuf 1995 die Videoskulptur Fifa Fucky (Abb.4). ${ }^{11}$ Die Arbeit wirkt auf den ersten Blick wie ein Triptychon. Auf einer von der Decke hängenden Konstruktion aus weißem Polyäthylen befindet sich ein in der Mitte geteilter Gipsabguss des bekannten spätarchaischen Sportlerreliefs im Nationalmuseum von Athen, auf dem sechs Athleten beim Ballspiel dargestellt sind. Zwischen den beiden Hälften ist ein um 90 Grad gekippter Fernsehapparat platziert. Auf dem Monitor, dessen Höhe der des Reliefs entspricht, läuft ein Videoband, das Bilder unterschiedlicher Herkunft von ballspielenden Männern und Frauen zeigt. Zu sehen sind zum Beispiel zwei nackte Frauen, deren Gesichter hinter weißen Masken verborgen sind. Sie verhalten sich defensiv, versuchen, den Fußbällen auszuweichen und sie abzuwehren, werden aber dennoch hart an Hals, Brust, Schenkel und Hüften getroffen. Die nackten weiblichen Körper wirken schutzlos und verletzlich und stehen damit in denkbar großem Kontrast zu den souverän agierenden männlichen Sportlern auf den Reliefs.

Die Relieffiguren und der Film sind formal aufeinander bezogen. Es hat den Anschein, als ob die antiken Sportler im nächsten Moment in den TV-Monitor und den Film hineinlaufen, als ob es sich um einen durchgehenden Fries handelt. Werfen oder schießen die antiken - männlichen - Sportler die Bälle, welche die Frauen treffen? Handelt es sich bei dieser Szene um eine Darstellung des Geschlechterkampfes? Ganz gewiss geht es um Existenzielles, um Sexualität, Stärke und Schwäche, Sieg und Niederlage, Angriff und Verteidigung. Der Titel Fifa Fucky enthält sowohl eine eindeutige sexuelle Konnotation als auch »einen ironischen Bezug [...] zu den Fernsehübertragungen der profanierten und kommerzialisierten Olympiaden heutiger Zeit $\ll{ }^{12}$ Das Weiß des Gipses und die Statik des Reliefs kontrastieren wirkungsvoll mit den farbigen, bewegten Bildern im Film, die von den Künstlern selbst inszeniert wurden. In der Videoskulptur von Marc Weis und Martin de Mattia erscheinen Antike und Gegenwart unmittelbar miteinander verbunden und dennoch weit voneinander entfernt, Kontinuitäten und Brüche werden gleichzeitig spürbar.

11 Vgl. Buhlmann (1996), 10, und Schneider (1998). Die Arbeit existiert in zwei Exemplaren, die sich im Suermondt-Ludwig-Museum, Aachen, und im Musée d'Art Moderne Grand-Duc Jean, Luxemburg, befinden. 1998 schufen die Künstler die (Nachfolge-)Arbeit Fucky Fight, abgebildet in Schneider (1998), 24f. Ich danke dem M + M Künstlerduo für seine Unterstützung.

12 Sven Thomas, in: TV-Kultur (1997), 375. 


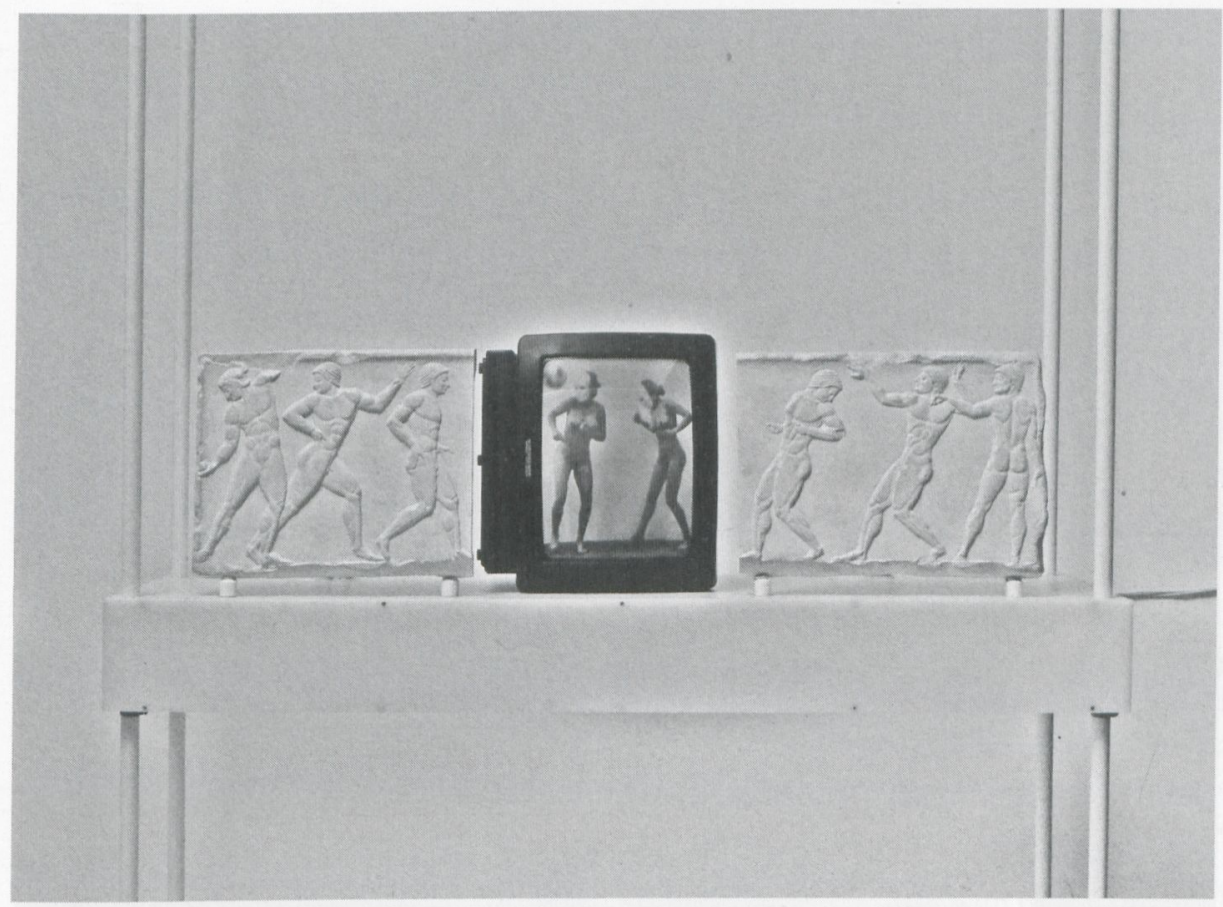

Abb. 4 M + M Künstlerduo (Marc Weis, Martin de Mattia), Fifa Fucky, 1995, Videoskulptur, 300 x 140 x $43 \mathrm{~cm}$, Aachen, Suermondt-Ludwig-Museum

\section{Von der ewigen Wiederkehr der Dinge: Liane Lang}

Der sogenannte Barberinische Faun, eine hellenistische Marmorskulptur aus dem 3. Jahrhundert v. Chr., gehört zu den berühmtesten Antiken der Glyptothek in München. Abb. 5 zeigt den Gipsabguss dieser Skulptur im Archäologischen Institut der Universität Heidelberg, frontal vor schwarzem Hintergrund aufgenommen. Doch der schlafende Satyr hat offensichtlich Besuch bekommen. Aus einem hellen Tuch, das um seinen Unterkörper drapiert ist, kommen zwei schlanke Beine, die zwischen den muskulösen Oberschenkeln des Satyrn nach unten hängen. Weiterhin sind zwei Unterarme zu sehen, die von hinten den Oberkörper umschlingen und sich zärtlich an seine Brust schmiegen. Es entsteht der Eindruck, als gehörten die Extremitäten zu einem hinter der Skulptur stehenden, vermutlich weiblichen Körper, doch bei genauerem Hinsehen zeigt sich, dass das anatomisch gar nicht möglich ist und außerdem die Gliedmaßen recht künstlich aussehen. Ein weiteres irritierendes Detail kommt hinzu: In der Öffnung unterhalb der linken Achsel des Fauns ist ein Mund zu erkennen, der 


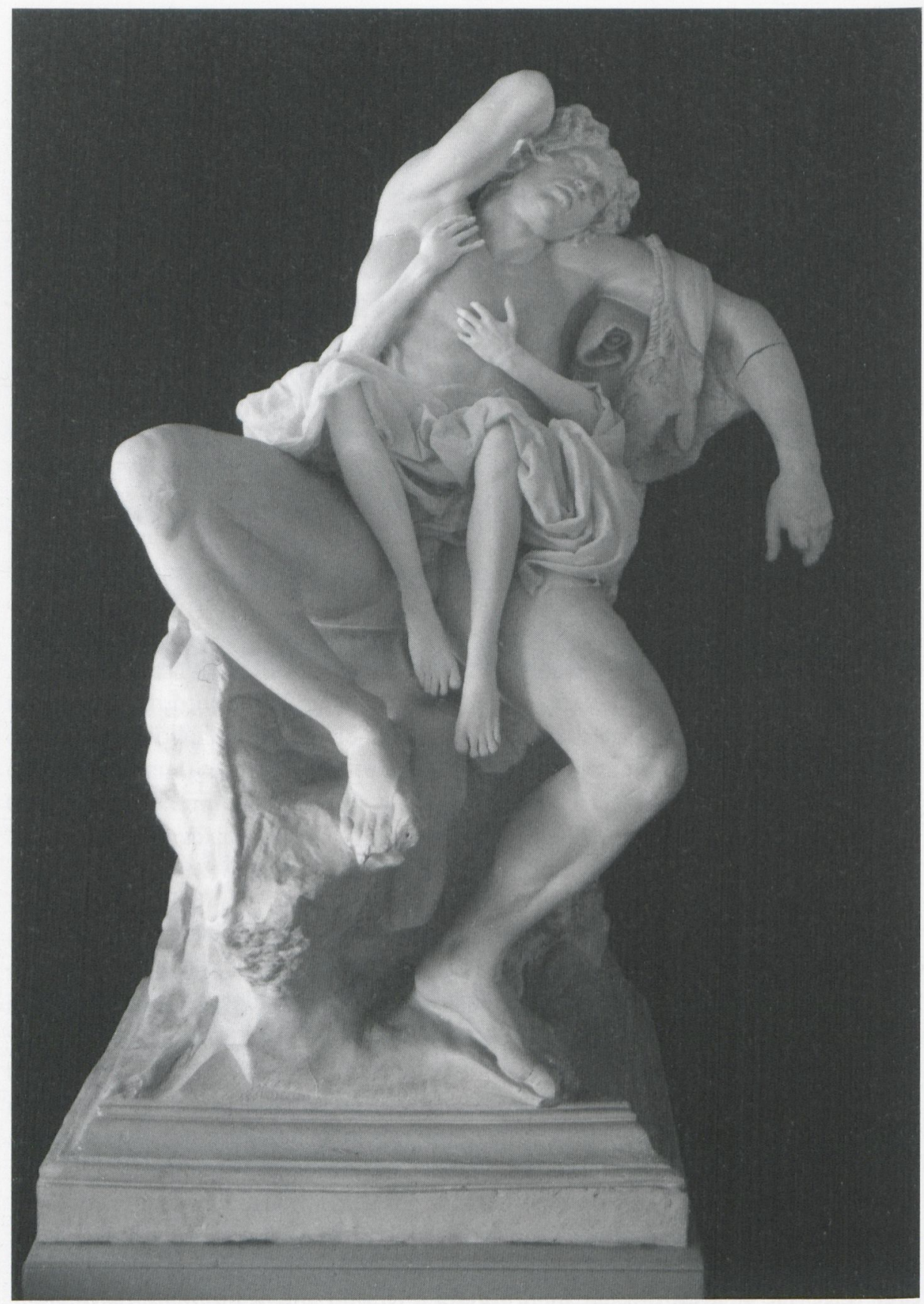

Abb. 5 Liane Lang, The Faun, 2007, C-Print, 150 x 125 cm, Besitz der Künstlerin 
wie zum Schrei geöffnet ist. Es handelt sich um die inszenierte Fotografie The Faun von Liane Lang aus dem Jahr 2007.

Liane Lang wurde 1973 in München geboren, studierte in Dublin und London und lebt in London. Die Film- und Fotokünstlerin arbeitet an der Schnittstelle von Skulptur und Fotografie. 2006/07 entstand die Fotoserie The Casts / Royal Academy Series. In der Gipsabgusssammlung der Royal Academy of Arts in London, an der die Künstlerin zu jener Zeit studierte, fügte sie Abgüssen antiker und klassischer Skulpturen künstliche, aus Latex, Wachs, Silikon oder Gummi modellierte Gliedmaßen hinzu. Dann fotografierte sie die so inszenierten und verfremdeten Gipse. 2007, anlässlich einer Einzelausstellung im Heidelberger Kunstverein, setzte Liane Lang diese Arbeit fort und schuf in der Gipsabgusssammlung des Archäologischen Instituts der Universität Heidelberg nach demselben Prinzip die Fotoserie Spectres, zu der The Faun gehört.

$\mathrm{Zu}$ ihrer Arbeit bemerkt die Künstlerin:

[...] In photographing the sculptures I wanted to re-animate them, invigorate their physical qualities, their presence as objects. Erotic curves and delicately weighted limbs interspersed with missing digits and the wear of centuries. [...] The antique sculptures had acquired a sense of abstraction through missing limbs and very worn faces, which I sought to exploit in re-composing them, interacting with my latex figures posing as living, breathing, flesh and blood.

In the series produced at the University of Heidelberg I worked with purely classical sculptures and I drained my figures of some of that life, making them pale and stony to merge and amend the shape of the sculptures they interfered with. Using echoes of mythology in constructing the narrative of the photograph, was an appealing process to me in different ways. The universality of mythological storytelling is so ubiquitous as to be almost inevitably a cliché. For the same reason however, its pertinence as a piece of narrative symbolism is universally understood. [...]

The fact that some antique sculpture has made it to us through so many changes of regime and religion, is the triumph of the object over its maker against all odds $[\ldots]]^{13}$

Die Aneignung der antiken Skulpturen erfolgt bei Lang auf zwei Ebenen: zum einen auf einer physischen Ebene, durch das Arrangieren und Verfremden der Gipsabgüsse mit künstlichen Gliedmaßen, zum anderen auf einer symbolischen Ebene, durch die fotografische Aufnahme. Es geht der Künstlerin, wie das Zitat deutlich macht, um eine »Reanimierung « der Skulpturen, um eine Belebung ihrer physischen Qualitäten als Objekte, was ihre zeitüberdauernde Präsenz unterstreicht. Liane Lang verlebendigt die Skulpturen. Ihre Fotografien erzählen Geschichten - Geschichten von Körpern, in deren Erscheinung vergangene Zeiten und Mythen nachhallen und die zugleich seltsam zeitenthoben und geisterhaft

13 Liane Lang, Thoughts on sculpture and animation, undatiertes Manuskript, von der Künstlerin am 23. November 2008 per E-Mail erhalten. Ich danke Liane Lang für diesen Text und für wertvolle Auskünfte. 
wirken. Dabei treibt die Künstlerin ein geistreiches Spiel mit der Wahrnehmung des Betrachters, der von dem, was er sieht, irritiert wird, sich das Ganze nicht recht zu erklären vermag. Antike und Gegenwart schieben sich förmlich ineinander, ohne zu einer wirklichen Symbiose zu kommen. Letztlich wirkt alles irgendwie künstlich, die ergänzten Körperteile ebenso wie der Gipsabguss, der am linken Unterarm eine deutlich sichtbare Bruchstelle aufweist und dem an der linken Hand einige Fingerglieder fehlen. Zu dieser Künstlichkeit passt auch die Inszenierung der Figur, die vor schwarzem Hintergrund freigestellt und somit entkontextualisiert ist. ${ }^{14}$ Liane Lang deckt die Künstlichkeit und Brüchigkeit unseres Antikenbildes auf und demonstriert zugleich dessen Wandelbarkeit, indem sie beispielsweise, wie in The Faun, die erotische Komponente der antiken Skulpturen betont. ${ }^{15}$

\section{Resümee}

Die vier besprochenen, zwischen 1975/76 und 2007 geschaffenen Werke verbindet eines: der Rekurs auf die klassische Antike durch die Einbeziehung von Gipsabgüssen, also mechanisch hergestellten Reproduktionen im Format 1:1. Die künstlerischen Strategien lassen sich als »Praktiken des Sekundären« beschreiben:

Als »Praktiken des Sekundären« sollen hier jene kulturellen und medialen Verfahren verstanden werden, die gezielt auf den Status des Vorgefundenen, des Nicht-Authentischen oder des Abgeleiteten ihres Gegenstands bzw. Materials setzen - oder aber derartige Zuschreibungen bewusst problematisieren. Es ist diese dezidierte Aneignung, die Praktiken des Sekundären von Produktionsweisen unterscheidet, die zwar notwendig auch auf Traditionsbestände zurückgreifen, dies jedoch - häufig unter den Vorzeichen von Originalität und Authentizität - unterschlagen, verdrängen oder zumindest nicht explizit ausweisen. ${ }^{16}$

Die Arbeiten gehören unterschiedlichen Gattungen an: Bei Kounellis handelt es sich um ein Objekt bzw. eine Assemblage, bei Paolini um eine Gruppe von Plastiken, beim M + M Künstlerduo um eine Videoskulptur und bei Liane Lang schließlich um eine Farbfotografie. Nicht nur in technischer Hinsicht, sondern auch im Hinblick auf die formale Verwendung des Gipsabgusses unterscheiden

14 Die entkontextualisierende fotografische Aufnahme von antiker (und moderner) Plastik vor schwarzem Hintergrund ist eine archäologische Konvention des 19. Jahrhunderts. Vgl. zur fotografischen Medialisierung den Beitrag von Stefanie Klamm in diesem Band, S.47-67.

15 An dieser Stelle sei auf die fiktiven Atelierinszenierungen des in Wien tätigen Künstlers Lois Renner verwiesen, in dessen Farbfotografien mehrfach der Barberinische Faun erscheint. Vgl. Lois Renner (2003) und von Rosen (2007).

16 Fehrmann/Linz/Schumacher/Weingart (2004), $7 \mathrm{f}$. 
sich die Arbeiten erheblich: Kounellis kombiniert zwei gefasste Fragmente von Gipsabgüssen; Paolini verwendet keine Fragmente, sondern ordnet zwei intakte Kopien ein- und derselben Figur in spezifischer Weise an, ohne weiter in die »Originale« einzugreifen, also ohne sie zu bearbeiten, zu verfremden; das M + M Künstlerduo ergänzt den in der Mitte geteilten Gipsabguss eines antiken Sportlerreliefs durch einen ins Hochformat gedrehten Fernsehapparat, auf dem ein Videoband läuft; und Liane Lang schließlich inszeniert einen Gipsabguss durch temporäre Hinzufügungen, denen die Fotografie Dauer verleiht.

Kounellis und Paolini sind vor dem Hintergrund einer Neubewertung des antiken Mythos in den 1970er Jahren auch und gerade in Italien zu sehen, die bei den Künstlern der »Arte povera « zu einer ausgeprägten Antikenrezeption führte. »Der griechische Götterhimmel«, schreibt Helmut Friedel hierzu, »wird zitiert, antike Bilder in klassizistischer Tradition werden aufgegriffen, Themen antiker Autoren geben den Stoff zu neuen Bildern ab. Die ausgeprägte Kenntnis dieser alten Vorstellungen sowie die alltägliche Präsenz der bildnerischen Überreste der Antike sind in Italien zudem ausschlaggebend dafür, daß es zu einer fast selbstverständlichen Neubearbeitung dieser Bilder kam. $\ll^{17}$ Während Kounellis der Antike melancholisch nachzutrauern scheint und sie nur in fragmentarischer Form bildnerisch verarbeitet, zitiert Paolini den antiken Kanon, um grundsätzliche Probleme der Kunst zu erörtern, wie das Verhältnis von Original und Kopie und das Sehen an sich.

Das M + M Künstlerduo und Liane Lang gehören jüngeren Künstlergenerationen an, die sich neuer Medien bedienen, der Videokunst und der Fotografie (wobei der Begriff des Originals im Hinblick auf Video und Fotografie seine eigene Problematik hat). Das jeweilige Medium trifft auf die klassische Skulptur, und gerade in dieser Konfrontation scheint für die Künstler ein besonderer Reiz zu liegen. Während beim M + M Künstlerduo zeitübergreifende, existenzielle Fragen behandelt werden, demonstriert Liane Lang die Künstlichkeit und Wandelbarkeit unseres Antikenbildes.

Konstitutiv für alle besprochenen Werke ist die Verwendung von Gipsabgüssen als bildnerisches Material. Das an sich ist schon bemerkenswert, weil diese traditionell nur als archäologisches oder akademisch-didaktisches Anschauungs- und Studienmaterial dienen und nicht als bildnerisches Primärmaterial. Hinzu kommt, dass der Gipsabguss in der Geschichte durchaus unterschiedliche Bewertungen erfahren hat. ${ }^{18}$ Nach langen Phasen großer Wertschätzung wuchs, vor allem in Künstlerkreisen und an den Kunstakademien, ab etwa Mitte des 19. Jahrhunderts die Kritik an Gipsabgüssen, die sich einerseits an ihrer materiellen wie auch künstlerischen Wertlosigkeit festmachte und ande-

17 Friedel (1988), $91 \mathrm{f}$.

18 Im Folgenden beziehe ich mich auf Cain (1995). 
rerseits vor dem Hintergrund der generellen Kritik an der normativen Vorbildhaftigkeit antiker Kunst zu sehen ist. Diese Tendenz verstärkte sich im Zuge der historischen Avantgardebewegungen seit dem Impressionismus.

Die generellen Zweifel am ideellen Vorbildcharakter antiker Kunst und erst recht der weißen Gipse wurden schließlich so stark, daß ihre ehemalige Wertschätzung seit den zwanziger Jahren [des 20. Jahrhunderts] ins Gegenteil gewendet werden konnte. Die Abgüsse galten nun als die blutleeren weißen Gespenster, denen nichts von der Aura der Originale eigen war. ${ }^{19}$

Das änderte sich in den späten 1960er Jahren erneut:

Erst eine im Kontext der Postmoderne kühl gestimmte neohistoristische Ästhetik sowie eine durch die technologische Entwicklung digitaler Technologie geschärfte Aufmerksamkeit für historische Speichermedien weckten erneut das Interesse für die Gipsabgüsse als Geschichtszeugen und auch eine neue Sensibilität für deren formale Qualitäten. ${ }^{20}$

Auch bei den vorgestellten Werkbeispielen werden die Gipskopien bzw. deren Fragmente als eigenständige, vollgültige Bildelemente verwendet, wobei die Antike indirekt, mittelbar, eben über das Medium des Abgusses, zitiert bzw. integriert wird. In den Fällen, in denen es sich um Abgüsse von römischen Kopien nach griechischen Originalen handelt, also in den Arbeiten von Jannis Kounellis und Giulio Paolini, haben wir es sogar mit der »zweite[n] Ableitung vom Original $\ll^{21} \mathrm{zu}$ tun.

Aus welcher Zeit stammen die antiken Originale? Kounellis, Paolini und Lang beziehen sich auf hellenistische Werke (bzw. römische Kopien nach hellenistischen Werken), M + M auf ein Relief aus der archaischen Zeit. Die Skulptur der griechischen Klassik des 5. Jahrhunderts, repräsentiert durch Polyklet, wird nicht ein einziges Mal ausgewählt. Ob man im Hinblick auf die in diesem Aufsatz nur an wenigen Exempla behandelte Kunst der letzten Jahrzehnte, in der antike Werke rezipiert werden, aber tatsächlich von einer »Entdeckung der Archaik« sprechen kann, die als »Grundzug der Antikenrezeption im 20. Jahrhundert ${ }^{22}$ beschrieben worden ist, darf zumindest bezweifelt werden.

Jannis Kounellis, Giulio Paolini, M + M und Liane Lang, die in ihrer Kunst allesamt konzeptuell arbeiten, nähern sich der Antike mit Respekt und Wertschätzung, also eher affirmativ denn kritisch oder gar aggressiv. In der Kunstgeschichte des 20. Jahrhunderts finden sich aber auch zahlreiche Beispiele, in denen die Antike negativ konnotiert und bisweilen bildnerisch regelrecht attackiert wird. Eine Fundgrube ist hier der Katalog zur Pariser Ausstellung

19 Ebd., 210.

20 Uppenkamp (2002), 110.

21 Bätzner (2000), 92.

22 Seidensticker/Vöhler (2001), VIII. 
D'après l'antique (2000/2001), in welchem u.a. folgende Kunstwerke reproduziert und besprochen sind, die allesamt ebenfalls Gipsabgüsse von Antiken verfremden: Venus de Milo aux tiroirs (1936) von Salvador Dalì, der aus dem Körper der Venus mehrere geöffnete Schubladen herausragen lässt; Tête de Venus otorhinologique (1964), ebenfalls von Salvador Dalì, der hier den Kopf der Venus von Milo mit einem Ohr auf der Nase und einer zweiten Nase anstelle des linken Ohres versieht; Roped Venus (1971) von Clive Barker, der einen Gipsabguss der Venus von Milo von den Füßen bis zur Brust mit einem dicken Seil eng umwickelt. ${ }^{23}$ Alle genannten Werke stellen die normative Vorbildhaftigkeit und die Unantastbarkeit antiker Klassiker in Frage und dekonstruieren auf diese Weise das an der Antike orientierte Schönheitsideal. Dessen Ende hatte bereits der italienische Schriftsteller F. T. Marinetti im ersten futuristischen Manifest postuliert, das am 20. Februar 1909 im Pariser »Figaro« erschien: »Wir erklären, daß sich die Herrlichkeit der Welt um eine neue Schönheit bereichert hat: die Schönheit der Geschwindigkeit. Ein Rennwagen [...] ist schöner als die $>$ Nike von Samothrake ${ }^{24}{ }^{24}$

Durch den Rekurs auf die Antike führen die vorgestellten Künstler in ihren Werken Diskurse über Gedächtnis und Erinnerung, über Modi der Repräsentation und über »Kunst über Kunst«. Da es jeweils auch um die Reflexion grundsätzlicher Fragen der Kunst geht - etwa des Verhältnisses von Antike und Moderne und von Original und Kopie, der Repräsentation der Geschlechter und medien- und gattungsspezifischer Aspekte -, möchte ich von »Metakunst« sprechen. Darunter verstehe ich »Kunst über Kunst« im doppelten Sinne: nämlich eine Kunst, die sich zum einen explizit auf ein bestimmtes Werk, einen Topos oder das institutionelle Umfeld der Kunstgeschichte bezieht und die zum anderen auf einer Metaebene, in einem bildnerischen Diskurs, Kunst thematisiert, sich also selbst reflektiert. Eine solche Metakunst gibt es erst seit etwa $1960 .^{25}$

Auch - oder gerade! - in Zeiten der vielbeklagten postmodernen Beliebigkeit scheint die Antike also nichts von ihrer Faszination verloren zu haben. Und das gilt nicht nur für Künstlerinnen und Künstler. So verknüpfte der Kunstwissenschaftler Roger M. Buergel die für ihn zentrale Frage nach der Aktualität der Moderne mit dem Verweis auf die Antike, indem er als eines der drei Leitmotive der von ihm geleiteten documenta XII die Frage formulierte: »Ist die Moderne unsere Antike? $\ll^{26}$

23 Vgl. D'après l'antique (2000), 464 (Nr. 259), 466 (Nr. 261), 484f. (Nr. 272a).

24 Zitiert nach dem Wiederabdruck der deutschen Übersetzung von Christa Baumgarth in Manifeste und Proklamationen der europäischen Avantgarde (1995), 5.

25 Vgl. hierzu Zuschlag (2002). Die Publikation der Habilitationsschrift des Verfassers zu diesem Thema befindet sich in Vorbereitung.

26 Zitiert nach Schöllhammer (2007). 


\begin{abstract}
Transformations of Antiquity in Contemporary Art

The essay examines the transformation of ancient Greek and Roman sculpture in contemporary sculpture, installation art, video sculpture and photography. Since the 1970s, artists of the Arte Povera and Concept Art movements like Giulio Paolini (born 1940) and Jannis Kounellis (born 1936) have been using plaster casts of antique sculptures in their collages, assemblages, sculptures and installations. Kounellis's Senza Titolo (1978) creates a mournful abbreviation of the human figure by utilising casts of a head and a hand taken from canonised classical statues, thereby evoking antique culture as a lost Arcadia. Paolini's Mimesi (1975/76), a combination of two plaster casts of the Venus Medici, focuses on the traditional role of imitation of nature and art, respectively, and the (postmodernist) problem of reproducibility, thus deliberately confounding the hierarchies of originals and copies.

The artist-duo M + M (Marc Weis, born 1965, and Martin de Mattia, born 1963) created the video sculpture Fifa-Fucky in 1995. Between two plaster copies of antique reliefs representing athletes, a television screen shows a videotape of people playing miscellaneous ballgames. The tension between the elements of the work is based on a dialogue between different concepts of gender and corporeality in ancient Greek and contemporary culture.

The last example considered is the work of the film and photo artist Liane Lang, who was born in Munich in 1973 and currently lives in London. Lang works at the interface between sculpture and photography in her two series The Casts / Royal Academy Series (2006/07) and Spectres (2007), taking reanimating photographs of plaster casts of antique sculptures estranged by artificial limbs.

The fact that many artists appropriate canonical sculptures of the European tradition (e.g. the Apollo Belvedere or the Venus Medici) and utilise plaster casts (bearing in mind their traditional role in the history of European art) challenges the comfortable assumption that archaic works of art dominate the $20^{\text {th }}$-century artistic approach to the heritage of antiquity.

The examples given are representative of an appreciation of antiquity. They speak of the relationship between the ancient and the contemporary, of the divide between an original and its copy, of memories and remembrance, and of modes of representation. Thus they are »art about art« (meta-art).
\end{abstract}




\section{Literatur}

Bätzner, Nike, Arte Povera. Zwischen Erinnerung und Ereignis: Giulio Paolini, Michelangelo Pistoletto, Jannis Kounellis, Nürnberg 2000.

Bann, Stephen, Jannis Kounellis, London 2003.

Berchtold, Maike, Gipsabguss und Original. Ein Beitrag zur Geschichte von Werturteilen, dargelegt am Beispiel des Bayerischen Nationalmuseums München und anderer Sammlungen des 19. Jahrhunderts, maschinenschriftliche Dissertation, Stuttgart 1987.

Buhlmann, Britta E., »Von Bildern, Abbildungen und Erinnerungen«, in: Junge Kunst Saar Ferngas Förderpreis 1996 (Katalog zur Ausstellung des Wilhelm-Hack-Museums Ludwigshafen am Rhein, 1. Juni bis 7. Juli 1996, der Brandenburgischen Kunstsammlungen Cottbus, 21. Juli bis 27. August 1996, und des Museums St. Wendel, 20. September bis 10. November 1996), Dillingen/Saar 1996, 9-12.

Cain, Hans Ulrich, »Gipsabgüsse. Zur Geschichte ihrer Wertschätzung «, in: Anzeiger des Germanischen Nationalmuseums und Berichte aus dem Forschungsinstitut für Realienkunde 1995, 200-215.

D'après l'antique (Katalog zur Ausstellung der Réunion des Musées Nationaux im Musée du Louvre, Paris, vom 16. Oktober 2000 bis 15. Januar 2001), éd. par Laurence Posselle, Paris 2000.

Didi-Huberman, Georges, Ähnlichkeit und Berührung. Archäologie, Anachronismus und Modernität des Abdrucks, Köln 1999.

Fehrmann, Gisela/Linz, Erika/Schumacher, Eckhard/Weingart, Brigitte, »Originalkopie. Praktiken des Sekundären - Eine Einleitung«, in: Originalkopie. Praktiken des Sekundären (Mediologie, Bd. 11), hg. v. dens., Köln 2004, 7-17.

Friedel, Helmut, »Giulio Paolini. Der doppelte Blick«, in: Der Traum des Orpheus. Mythologie in der italienischen Gegenwartskunst 1967 bis 1984 (Katalog zur Ausstellung der Städtischen Galerie im Lenbachhaus München, 16. Mai bis 1. Juli 1984), hg. v. dems., München 1984, 72-74.

Friedel, Helmut, »Imago Memoriae. Themen, Motive und Vorbilder aus der klassischen Antike in der italienischen Kunst der siebziger und achtziger Jahre«, in: Mythos Italien - Wintermärchen Deutschland. Die italienische Moderne und ihr Dialog mit Deutschland (Katalog zur Ausstellung der Bayerischen Staatsgemäldesammlungen München und der Ausstellungsleitung Haus der Kunst München e. V., 24. März bis 29. Mai 1988), hg. v. Carla Schulz-Hoffmann, München 1988, 91-96.

Krüger, Reto, Nach der Antike. Studien zur Antikenrezeption in der bildenden Kunst seit 1967 (Schriftenreihe des Instituts für Kunst- und Designwissenschaften [IKUD] der Universität Essen-Duisburg, 9), Essen 2004.

Ladendorf, Heinz, Antikenstudium und Antikenkopie. Vorarbeiten zu einer Darstellung ihrer Bedeutung in der mittelalterlichen und neueren Zeit (Abhandlungen der Sächsischen Akademie der Wissenschaften zu Leipzig, Philologisch-historische Klasse, 46, H. 2), 2. erweiterte Aufl., Berlin 1958.

Lois Renner. Bilder 1991-2002, hg. v. Maribel Königer, Ostfildern-Ruit 2003.

Manifeste und Proklamationen der europäischen Avantgarde (1909-1938), hg. v. Wolfgang Asholt/Walter Fähnders, Stuttgart/Weimar 1995.

Melancholie. Genie und Wahnsinn in der Kunst (Katalog zur Ausstellung der Réunion des Musées Nationaux in den Galeries nationales du Grand Palais, Paris, 10. Oktober 2005 bis 16. Januar 2006, und der Neuen Nationalgalerie, Staatliche Museen zu Berlin, 17. Februar bis 7. Mai 2006), hg. v. Jean Clair, Ostfildern-Ruit 2005. 
Mythos Italien - Wintermärchen Deutschland. Die italienische Moderne und ihr Dialog mit Deutschland (Katalog zur Ausstellung der Bayerischen Staatsgemäldesammlungen München und der Ausstellungsleitung Haus der Kunst München e. V., 24. März bis 29. Mai 1988), hg. v. Carla Schulz-Hoffmann, München 1988.

Rosen, Valeska von, » 'Lieber Maler-Fürst als Foto-Graf $\prec$ Lois Renners fotografische Inszenierungen der Gattungen im Atelier«, in: Im Agon der Künste. Paragonales Denken, ästhetische Praxis und die Diversität der Sinne, hg. v. Hannah Baader/Ulrike Müller Hofstede/Kristine Patz/Nicola Suthor, München 2007, 542-562.

Schneider, Ulrich, »M+M: Antike live«, in: $M+M$ ein-aus (Katalog zur Ausstellung der Museen der Stadt Aachen, Suermondt-Ludwig-Museum, 1998), München 1998, 3 f.

Schöllhammer, Georg (Bearb.), Documenta Magazine No. 1, 2007. Modernity?, Köln 2007.

Seidensticker, Bernd/Vöhler, Martin, »Vorwort«, in: Urgeschichten der Moderne. Die Antike im 20. Jahrhundert, hg. v. dens., Stuttgart/Weimar 2001, VII-X.

Stabenow, Cornelia, »Jannis Kounellis«, in: Mythos Italien - Wintermärchen Deutschland. Die italienische Moderne und ihr Dialog mit Deutschland (Katalog zur Ausstellung der Bayerischen Staatsgemäldesammlungen München und der Ausstellungsleitung Haus der Kunst München e. V., 24. März bis 29. Mai 1988), hg. v. Carla Schulz-Hoffmann, München 1988, 304.

TV-Kultur. Das Fernsehen in der Kunst seit 1879, hg. v. Wulf Herzogenrath/Thomas W. Gaehtgens/Sven Thomas/Peter Hoenisch, Dresden 1997.

Unterdörfer, Michaela, Die Rezeption der Antike in der Postmoderne. Der Gipsabguss in der italienischen Kunst der 70er und 80er Jahre, Weimar 1998.

Uppenkamp, Bettina, »Gips«, in: Lexikon des künstlerischen Materials. Werkstoffe der modernen Kunst von Abfall bis Zinn, hg. v. Monika Wagner/Dietmar Rübel/Sebastian Hackenschmidt, München 2002, 106-113.

Zuschlag, Christoph, »Vom Kunstzitat zur Metakunst. Kunst über Kunst im 20. Jahrhundert«, in: Wettstreit der Künste. Malerei und Skulptur von Dürer bis Daumier (Katalog zur Ausstellung im Haus der Kunst München, 1. Februar bis 5. Mai 2002, und im Wallraf-Richartz-Museum - Fondation Corboud Köln, 25. Mai bis 25. August 2002), hg. v. Ekkehard Mai/Kurt Wettengl, Wolfratshausen 2002, 171-189.

\section{Abbildungsnachweis}

Abb. 1: Fotografie Claudia Branca. - Abb.2: (C) Jannis Kounellis, Fotografie Gudrun Gewecke. - Abb.3: (C) Giulio Paolini. - Abb.4: (C) VG Bild-Kunst, Bonn 2009, Fotografie Wilfried Petri. - Abb. 5: (c) Liane Lang. 